\title{
基于星载被动微波遥感的青藏高原湖冰物候监测方法
}

\author{
邱玉宝 ${ }^{1}$, 王星星 ${ }^{1,2}$, 阮永俭 ${ }^{1,3}$, 解鹏飞 ${ }^{1}$, 钟 钰 $^{1,2}$, 杨素萍 ${ }^{1}$ \\ (1: 中国科学院遥感与数字地球研究所数字地球重点实验室, 北京 100094) \\ (2: 江西理工大学建筑与测绘工程学院, 赣州 341000) \\ (3: 中山大学地理科学与规划学院,广州 510000)
}

\begin{abstract}
摘 要: 湖冰物候影响着区域及全球气候, 是全球变化的敏感因子, 青藏高原湖泊众多, 冻融现场监测数据缺乏, 而微波 具有对冰水相变敏感、时间分辨率高、历史存档数据长等特点, 这对于长时间序列湖冰物候研究具有重要意义. 然而, 被 动微波遥感空间分辨率低、湖泊亮温的精准定位难. 论文通过获取 AMSR-E/Aqua 和 AMSR-2/Gcom-W1 的亮温数据, 构建 了基于轨道亮温数据的阈值判别法, 通过对青藏高原不同区域和不同大小的青海湖、色林错、哈拉湖以及阿其克库勒湖 进行测试研究: 与青海湖现场观测对比, 湖泊完全冻结日期与开始融化日期最大误差小于 3 天; 与无云光学遥感判别结果 相比, 4 个湖泊的冻融参数误差为 $2 \sim 4$ 天. 结果表明,被动微波轨道亮温数据可实现青藏高原地区亚像元级中大型湖泊 冻融信息的获取,历史卫星资料可为湖冰物候的监测提供重要的支撑.
\end{abstract}

关键词: 湖冰物候监测; 星载被动微波遥感; 青藏高原; 阈值法

\section{Passive microwave remote sensing of lake freeze-thawing over Qinghai-Tibet Plateau}

QIU Yubao ${ }^{1}$, WANG Xingxing ${ }^{1,2}$, RUAN Yongjian ${ }^{1,3}$, XIE Pengfei ${ }^{1}$, ZHONG Yu ${ }^{1,2}$ \& YANG Suping ${ }^{1}$

(1: Laboratory of Digital Earth Science, Institute of Remote Sensing and Digital Earth, Chinese Academy of Sciences, Beijing 100094 , P.R.China)

(2: School of Architectural and Surveying and Mapping Engineering, Jiangxi University of Science and Technology, Ganzhou 341000, P.R.China)

(3: School of Geography and Planning, Sun Yat-Sen University, Guangzhou 510000, P.R. China)

Abstract: The freezing of the lake is one of the sensitive factors of global climate change, which affects regional and global climate. There are many lakes in the Qinghai-Tibet Plateau, however, the freeze-thawing ground monitoring data is very seriously lacking. The microwave has the characteristics of water phase sensitivity, high temporal resolution and long historical data, which are of great value for the monitoring of long-term lake ice phenology. However, the passive microwave has a low spatial resolution, and the brightness temperature of the lake has the challenge of positioning accuracy. The paper obtained the orbital brightness temperature data of AMSR-E/2 from the Aqua and Gcom-W1 satellite, and constructed the method of threshold freeze-thawing, which were based on the orbital brightness temperature data. These two methods were tested in different regions and sizes of lakes in the Qinghai-Tibet Plateau (Lake Qinghai, Siling Co, Lake Hala and Lake Aqikekule). The results showed that the maximum error of the freeze-up end date and the break-up start date were less than 3 days in comparison with the ground observation of Lake Qinghai, and compared with the results of the non-cloud optical remote sensing test, the parameters of the freeze-thawing parameters of the four lakes were 2-4 days. The study suggests that based on the brightness temperature data of passive microwave orbit, it is possible to achieve the acquisition of freeze-thawing information of sub-pixel large and medium-sized lakes in the Qinghai-Tibet Plateau, and using historical satellite data can provide an important reference for the monitoring of lake ice.

Keywords: Lake ice phenology; passive microwave remote sensing; Qinghai-Tibet Plateau; threshold method

* 中国科学院 A 类战略性先导科技专项 (XDA19070201)、中国科学院国际合作局对外合作重点项目 (131C11KYSB20160061) 和国家自然科学基金项目(41371351) 联合资助. 2017-08-21 收稿; 2018-01-04 收修改 稿. 邱玉宝(1978 ), 男,博士,副研究员;E-mail : qiuyb@ radi.ac.cn. 
全球气候变化是地球系统过程中的重要内容, 近年来, 地表气温、湖泊和海洋温度呈升高趋势 ${ }^{[1-2]}$, 直接 导致湖冰解冻提前、积雪消融、冰川退缩等现象加速发生 ${ }^{[3-4]}$. 湖冰是全球陆地淡水冰的主要组成部分, 其冻 融变化与气温、降水等气候参数关系密切, 湖冰封冻/解冻日期的变化会造成区域下垫面改变, 影响反照率 等关键参数, 从而对大气环流造成影响 ${ }^{[5]}$, 并通过水循环等机制对区域甚至全球的气候系统形成反馈和 影响 ${ }^{6]}$.

位于亚洲中部的青藏高原地区被认为是地球上对气候变化最敏感的区域之一 ${ }^{[7-9]}$, 由喜马拉雅山、昆仑 山、横断山、祁连山和天山等山脉及高原地区组成, 海拔范围在 $2000 \sim 8844 \mathrm{~m}$ 之间, 平均海拔约为 $4046 \mathrm{~m}$. 青 藏高原地区湖泊密集, 大小湖泊星罗棋布, 湖泊总面积约 $43065.97 \mathrm{~km}^{2}$, 面积大于 $1 \mathrm{~km}^{2}$ 的湖泊约有 1200 个, 是全球湖泊最为密集的区域之一 ${ }^{[10-12]}$. 青藏高原地区地理环境恶劣, 对湖泊综合观测的站点数量稀少, 地面 观测数据难以获取, 有关湖泊的研究主要集中在湖泊面积和水位变化等方面 ${ }^{[13-18]}$. 尽管有关湖冰物候变化 的研究在青藏高原及全球变化尺度上涉及较少 ${ }^{[19]}$, 但湖冰物候可真实反映各个湖泊所处的区域在不同时间 节点的气候变化信息及其变化趋势 ${ }^{[20]}$, 具有重要的气候学意义.

在实地监测缺失的情况下,遥感在湖冰物候信息获取方面有重要作用, 现有研究主要利用光学遥感及 微波遥感数据. 已有较多研究实现了利用光学遥感对湖冰监测, 如陈贤章等 ${ }^{[21]}$ 利用 AVHRR 的 $1 、 2$ 和 4 通 道数据监测青海湖获得 1993-1994 年湖水冻结情况; 殷青军等 ${ }^{[22]}$ 利用 MODIS1、2 波段来鉴别湖冰变化; Kropácek 等 ${ }^{[23]}$ 利用 8 天无云/少云积雪产品 MYD10A2(The MODIS Aqua Snow Cover 8-Day L3 Global $500 \mathrm{~m}$ Grid) 获得湖冰物候变化数据集; 姚晓军等 ${ }^{[24]}$ 利用 MOD09GA (MODIS/Terra Surface Reflectance Daily L2G Global $1 \mathrm{~km}$ and $500 \mathrm{~m}$ ) 数据实现了可可西里湖群的监测; 勾鹏等 ${ }^{[25]}$ 利用 MODIS 反射率数据获取纳木错湖 冰冻融数据集等. 但青藏高原地区云量覆盖研究表明 ${ }^{[26]}$, 在积雪季节, 研究区大部分地区云覆盖天数在 50 100 天, 个别区域云盖甚至大于 150 天, 云盖情况严重, 平均每天云覆盖占 $45 \%$, 利用光学传感器监测湖泊由 于云污染, 存在数据缺失的严重问题 ${ }^{[23-24,27]}$, 如 Kropácek 等 ${ }^{[23]}$ 所监测的湖冰物候, 其误差可达 8 天甚至 16 天. 显然, 湖冰物候变化快、对时间分辨率要求高的特点使其对光学遥感云污染所造成的数据缺失提出了 挑战.

而被动微波辐射计数据, 如 SSM/I(1987 年至今)、AMSR-E (2002-2011 年)、MWRI( 2010 年至今) 和 AMSR-2 (2012 年至今), 受大气状况影响较小, 对冰、水相变敏感, 时间分辨率高 (青藏高原地区的重访周期 1 2 天), 历史数据充足, 对湖冰物候监测具有重要意义. 在青藏高原区域利用被动微波辐射计监测湖冰物 候的有车涛等 ${ }^{[28]}$ 和陶安琪 ${ }^{[29]}$ 等, 主要利用重采样 EASE-Grid 亮温数据进行青藏高原地区两个大型湖泊(青 海湖与纳木错) 的湖冰物候判别方法研究. AMSR-E 的 EASE-Grid 格式 $18.7 \mathrm{GHz}$ 的亮温数据分辨率为 $25 \mathrm{~km} \times$ $25 \mathrm{~km}$, 是由瞬时视场角 (IFOV) 为 $27 \mathrm{~km} \times 16 \mathrm{~km}$ 轨道亮温数据经重采样、转投影等处理获得. AMSR-E 的轨 道亮温数据的定位误差约为 $5 \sim 7 \mathrm{~km}$, 而 EASE-Grid 亮温数据, 在投影转换的过程中带来一定的位置定位及 亮温的平滑误差 ${ }^{[30]}$, 会对位置要求苛刻的湖冰监测结果造成较大的影响, 其所能准确监测的湖泊数量大大 减少.

针对上述问题, 本研究选取时间上连续的 3 个传感器 AMSR-E (2002- 2011 年)、MWRI( 2010 年至今) 和 AMSR-2 (2012 年至今) $18.7 \mathrm{GHz}$ 垂直极化的轨道( Swath) 亮温数据二级产品, 通过获取青藏高原地区 4 个典型湖泊 (青海湖、色林错、哈拉湖及阿其克库勒湖) 距离湖泊中心点最邻近的像元亮温值, 并改进常用的 最大值、最小值搜索法对湖冰冻融参数进行提取, 发展了能够适用于青藏高原地区中大型湖泊 (微波亚像元 $350 \mathrm{~km}^{2}$ 以上,如阿其克库勒湖) 的湖冰物候微波监测方法.

\section{1 研究区概况}

青藏高原为高寒气候, 冰川、积雪及湖泊覆盖面积广, 该区的气候对局部气候甚至全球气候变化都有显 著影响, 被誉为 “全球气候变化的驱动与放大器” ${ }^{[31]}$. 湖泊冻融过程与外界能量交换过程、其周边的气候变 化有着密切的关系 ${ }^{[32]}$, 无人干扰的青藏高原湖冰冻融特征是气候变化研究的重要指标 ${ }^{[23]}$.

研究选取了青藏高原东北部、青藏高原北部、青藏高原腹部 3 处不同区域 4 个湖泊作为数据获取和冻融 判别方法研究的测试对象 (图 1). 且所选湖泊的面积具有明显差别, 其中大型湖泊青海湖面积约为 4449.7 
$\mathrm{km}^{2}$, 色林错约为 $1640.9 \mathrm{~km}^{2}$, 而相对小型湖泊哈拉湖面积为 $594.9 \mathrm{~km}^{2}$, 阿其克库勒湖面积 $355.6 \mathrm{~km}^{2[33]}$, 为 AMSR-E 瞬时视场(IFOV) 面积的 $82 \%$.

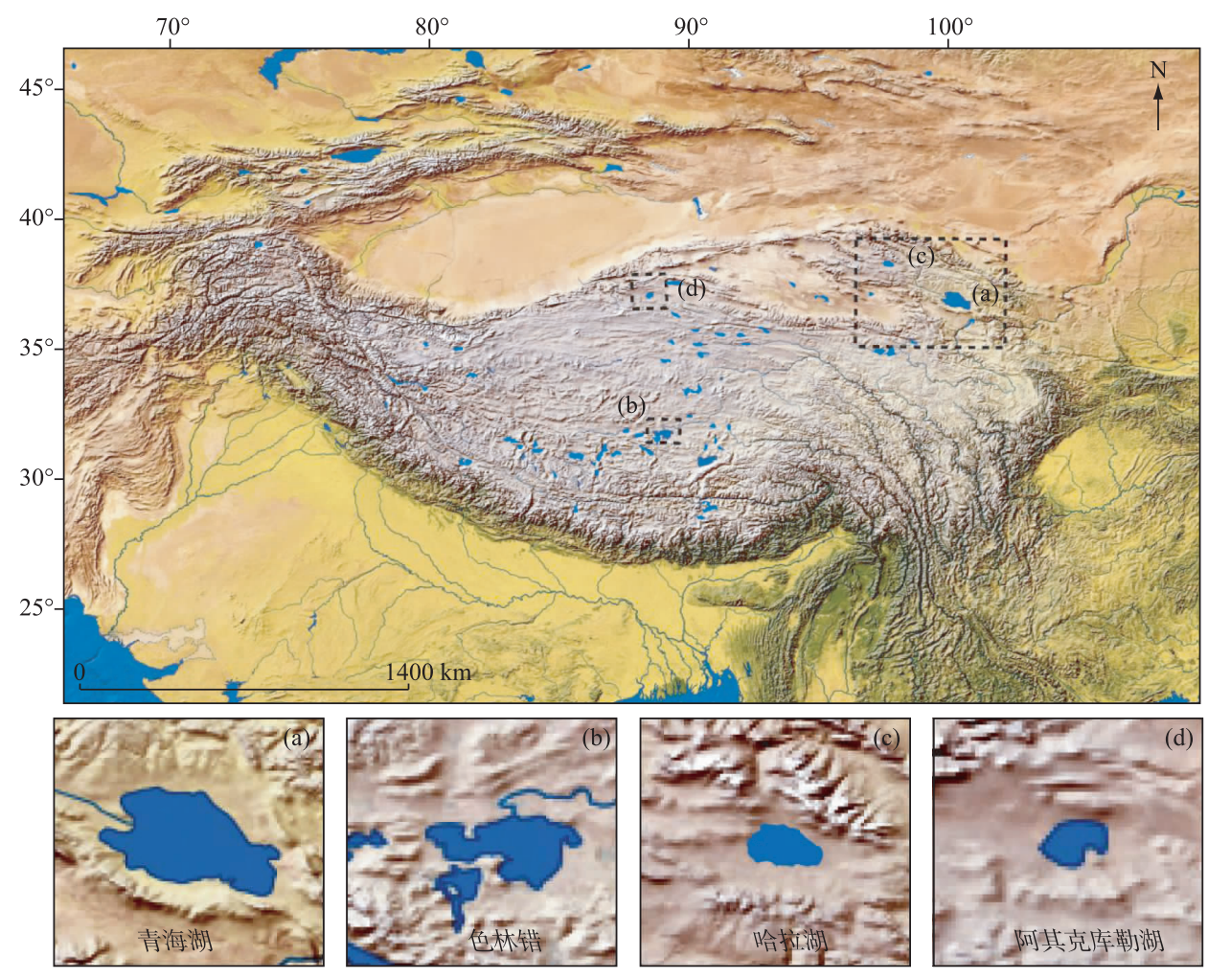

图 1 试验所选 4 个不同大小湖泊的分布

Fig.1 Distribution of selected lakes over Qinghai-Tibet Plateau

\section{2 数据与方法}

\section{1 星载微波辐射计数据}

大量微波辐射计自 1970s 后相继升空, 并获取了一系列长时间序列高质量、高时间分辨率的对地观测数 据, 为遥感监测湖冰物候长时间序列研究提供了基础. 研究所用传感器以 AMSR-E ( $2002-2011$ 年) 与 AMSR-2 (2012 年至今) 为主, 由于 AMSR-E 与 AMSR-2 两个不同的传感器在时间上未能衔接, 导致 2011 年 10 月 4 日一-2012 年 7 月 2 日数据缺失,为此利用国产风云卫星 MWRI( 2010 年至今) 进行数据信息补缺. 所 选择的被动微波数据 AMSR-E (Level 2A)、AMSR-2(Level 1R) 与 MWRI( Level 2A) 的不同频率的成像仪通道 都具有垂直极化与水平极化两种成像方式. 其中 AMSR-E 有 12 个成像仪通道, 频率分别为 $6.9 、 10.65 、 18.7$ 、 $23.8 、 36.5$ 和 $89.0 \mathrm{GHz}$, AMSR-2 有 14 个成像仪通道,频率分别为 $6.9 、 7.3 、 10.65 、 18.7 、 23.8 、 36.5$ 和 89.0 $\mathrm{GHz}$; 而 MWRI 的 10 个成像仪通道除 $6.9 \mathrm{GHz}$ 外, 与 AMSR-E 辐射计的频率设置完全一样, 其数据也开展了 交叉定标研究, 具有较好的一致性 ${ }^{[34-35]}$, 冰和水在 $18.7 \mathrm{GHz}$ 通道 $\mathrm{V}$ 极化的发射率差异显著, 所以研究选用 $18.7 \mathrm{GHz}$ 的 $\mathrm{V}$ 极化数据.

\section{2 湖泊亮温数据预处理和提取}

为了获得能表征湖泊的亮温值, 论文选用中高分辨影像 Landsat TM 辅助确定湖中心点位置, 再以该中 心获取其最邻近像元的亮温值. 在获得长时间序列的湖泊亮温值后, 由于被动微波辐射计条带覆盖不足, 会 存在 $1 \sim 2$ 天数据缺失情况, 则通过线性插值法对缺失日期的亮温进行插值. 针对湖泊亮温受外界局部环境 
影响所致的噪声, 采用中值滤波对湖泊亮温进行去噪等预处理, 在滤除噪声的同时, 可保护信号的边缘特 征,使之不被模糊. 虽然不同传感器有不同的亮温差异,但湖冰物候是通过获取亮温信号突变点的日期来判 别,所以这种差异对算法的影响较小 ${ }^{[36]}$.

在预处理之后,采用最邻近法动态获取湖泊亮温值(图 2): 通过在 Landsat 上选取离岸等距离的点为湖 泊中心点 $\mathrm{O}(X, Y)$, 以中心 $\mathrm{O}$ 经纬度增加和减少 $0.125^{\circ}$ 获得覆盖范围的 4 个顶点. 在获取 Swath 数据亮温值 时, 首先舍弃没有落在红色矩形内的亮温点, 当落在矩形框内只有一个点时, 所获取的点就是所求点. 若落 在红色矩形中的亮温点多于一个以上, 则选取距离中心点 $\mathrm{O}(X, Y)$ 最近的点 $\left(X_{\min }, Y_{\text {min }}\right)$, 其求取公式为:

$$
\sqrt{\left(X-X_{\min }\right)^{2}+\left(Y-Y_{\min }\right)^{2}} \leqslant \sqrt{\left(X-X_{i}\right)^{2}+\left(Y-Y_{i}\right)^{2}}
$$

$\left(X_{i}, Y_{i}\right)$ 表示落在红色矩形框内的任意一点.

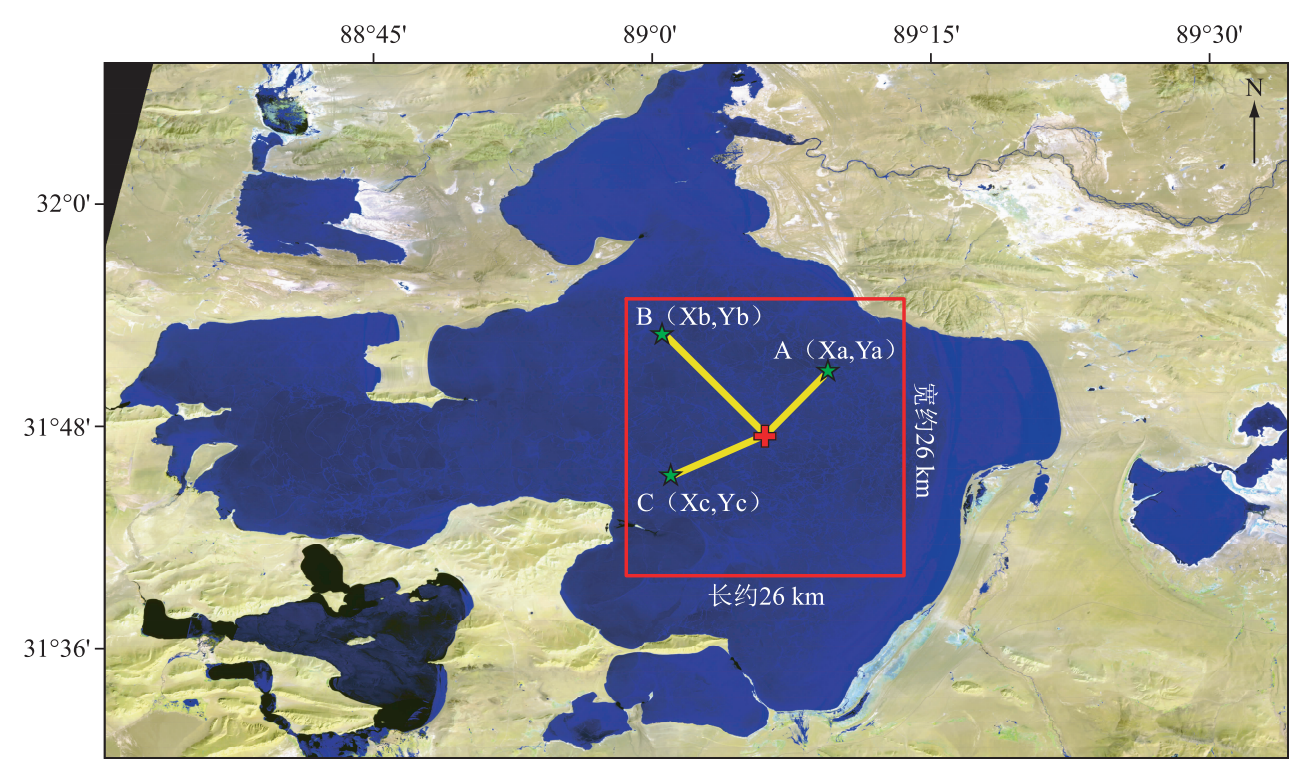

图 2 色林错湖泊亮温获取示意图

Fig.2 The example to obtain the lake's TB of Siling Co

通过上述数据预处理步骤获得长时间的湖泊亮温值, 以其中较小且比较规则的湖泊哈拉湖为例, 3 个传 感器的湖泊信号位置分布如图 3. 由于哈拉湖湖泊形状比较规则, 其 AMSR-E、AMSR-2 坐标点基本处于湖泊 中心附近, 且离湖岸有一定的距离, 可以获得比较 “纯净” 的湖泊亮温值. 而 MWRI 由于分辨率 (瞬时视场角 IFOV) 为 $30 \mathrm{~km} \times 50 \mathrm{~km}$, 其所获得接近湖泊外部范围的湖泊亮温值有较多的湖岸辐射污染, 本文对此污染只 能作为误差处理. 从图 3 可知, AMSR-2 因精度相对高, 其位置动态范围小, 所获取的亮温可清晰地反映湖泊 亮温的变化信息, 3 个传感器 $18.7 \mathrm{~V}$ 曲线以 AMSR-2 的亮温值最为理想, 而 AMSR-E 的亮温曲线有少量噪 声, MWRI 有较多的噪声. 因此, 对于湖冰物候研究, 以 AMSR-E 与 AMSR-2 数据为主, 而 MWRI 数据仅做补 充,应用于青海湖、色林错冻融监测.

\section{3 辅助和验证数据}

选择青海湖水文站 2002-2006 年地面观测的湖冰冻融数据和 2002-2015 年青藏高原 MODIS 逐日无 云积雪面积数据集作为验证的辅助数据. 通过文献获得青海湖湖冰冻融水文站数据 ${ }^{[28]}$, 青藏高原 MODIS 逐 日无云积雪面积数据集来源于 Science Data Bank, 该数据集是基于 MOD10A1 与 MYD10A1 (MODIS/Terra Snow Cover Daily L3 Global $500 \mathrm{~m}$ Grid) 经过去云算法处理的青藏高原每日无云的积雪产品 ${ }^{[37]}$. 该产品的地 物分类种类包括 “陆地、雪、湖泊、湖冰和湖泊不确定” 5 大类别, 可通过较高分辨率的 MODIS 逐日无云积雪 产品的湖水和湖冰变化情况来对比验证利用低分辨率的被动微波辐射数据提取的湖冰物候信息. 


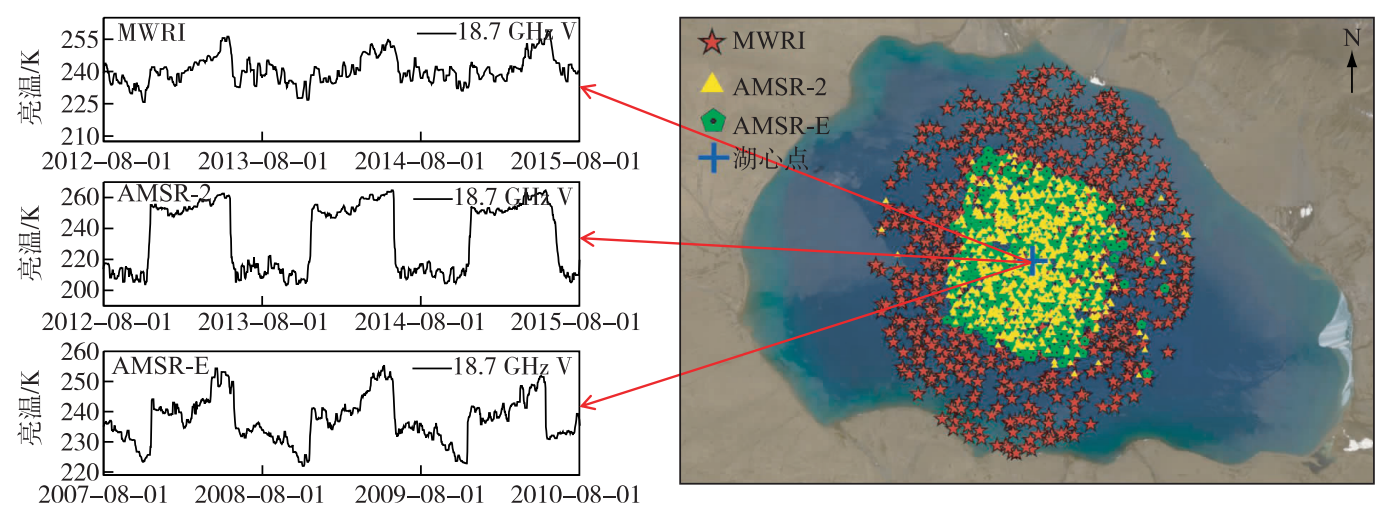

图 33 种传感器在哈拉湖的点值分布

Fig.3 Distribution of the TB's points of three sensors in Lake Hala

\section{4 冻融监测方法}

2.4.1 湖冰物候参数定义 湖冰物候的主要监测指标分别为 ${ }^{[23-24]}$ : 开始冻结 (freeze-up start, FUS)、完全冻结 (freeze-up end, FUE)、开始融化 (break-up start, BUS) 和完全融化 (break-up end, BUE). 当湖泊第 1 次出现能 够被监测到湖冰, 定义为湖冰开始冻结; 当湖泊第 1 次出现连续、完全覆盖湖面的稳定湖冰, 定义为湖冰完 全冻结; 当湖面冰层出现裂缝, 暴露出开放水体, 且不会再被重冻结, 定义为湖冰开始融化; 当湖水完全暴露 在空气中, 且不再有冰出现, 定义为湖冰完全融化.

2.4 .2 湖泊亮温曲线变化分析 在湖泊冻结期, 由水转换成冰时, 湖泊区域发射率提高, 湖泊亮温值会骤然升 高; 而在湖冰融化期, 冰转换成水时湖泊区域发射率降低, 湖泊亮温值会骤然下降 ${ }^{[28-29]}$. 但是不同湖泊的自 身要素 (面积大小、形状及湖泊的盐度等特性) 和所处的自然环境不同,其冻融时亮温变化幅度的升高与下 降也略有不同. 由图 4 可知, 青海湖与色林错的湖泊亮温信号变化趋势明显, 其变化与理论相符; 而面积较 小的哈拉湖与阿其克库勒湖则在非冰冻期出现信号波动现象, 主要来源于湖泊的温度和其他天气现象变 化; 而在封冻期出现双峰波动现象, 这可能是受到湖冰厚度变化 ${ }^{[28,38]}$ 以及湖泊周边环境变化 (如风、雪) 的影 响 ${ }^{[28]}$,如风对小湖湖冰的形成有抑制作用,使得已经冻结的湖泊再次解冻 ${ }^{[39]}$.

2.4 .3 冻融判别方法 由于不同湖泊湖冰冻融突变节点的亮温值不同, 难以直接利用统一的國值法获取冻融 参数 ${ }^{[25]}$. 人工目视判读精度虽高, 但数据量大且与解译人员的专业和经验有关. 在过去的研究中 ${ }^{[28-29]}$, 通过 搜索亮温差确定湖泊冻结与消融日期,但该算法是基于比较规则的湖泊、像元 “纯净” 的亮温时序数据,冻融 参数的判定很容易受到极值的影响, 而且仍需加人大量的人工解译 ${ }^{[29]}$, 该类方法对有混合像元 (或亚像元 级) 的大量湖泊进行长时间序列的湖冰物候获取存在着较大的提升空间. 为了减小极值的影响且更加快速 获取物候参数过程, 引人阈值法, 通过对亮温差值限定时间窗口, 并累加判断, 可以减少人工判读的主观误 差, 更快速精准地获取湖冰物候参数. 技术流程见图 5,详细算法描述如下:

a) FUE、BUS 湖冰冻融参数获取

传感器探测到的湖泊亮温值受冰、水介电特性影响的同时也受湖泊的形状、大小、含盐量等属性的影 响, 导致不同湖泊的亮温节点变异值不同, 4 天最大值、最小值搜索法可以实现湖冰物候参数的初步判别, 引 人阈值判别法可以更进一步地实现 FUE 与 BUS 的获取.

4 天最大值与最小值搜索法是通过求取一个冻融周期内 (如 2003 年 8 月 1 日一-2004 年 7 月 31 日为 1 个 冻融周期) 每天与之前 3 天的亮温平均值、与之后 3 天的亮温平均值的差值, 进一步扩大湖泊亮温曲线在冰 水相变时的差异, 进而求出初步的 FUE 与 BUS 参数. 4 天最大值与最小值搜索法数学表达公式如下 ${ }^{[27]}$, 其 获取结果示例如图 6 所示:

$$
\begin{aligned}
& F U E=\operatorname{Min}\left(\left(B T_{i}+B T_{i-1}+B T_{i-2}+B T_{i-3}\right) / 4-\left(B T_{i}+B T_{i+1}+B T_{i+2}+B T_{i+3}\right) / 4\right) \\
& B U S=\operatorname{Max}\left(\left(B T_{i}+B T_{i-1}+B T_{i-2}+B T_{i-3}\right) / 4-\left(B T_{i}+B T_{i+1}+B T_{i+2}+B T_{i+3}\right) / 4\right)
\end{aligned}
$$



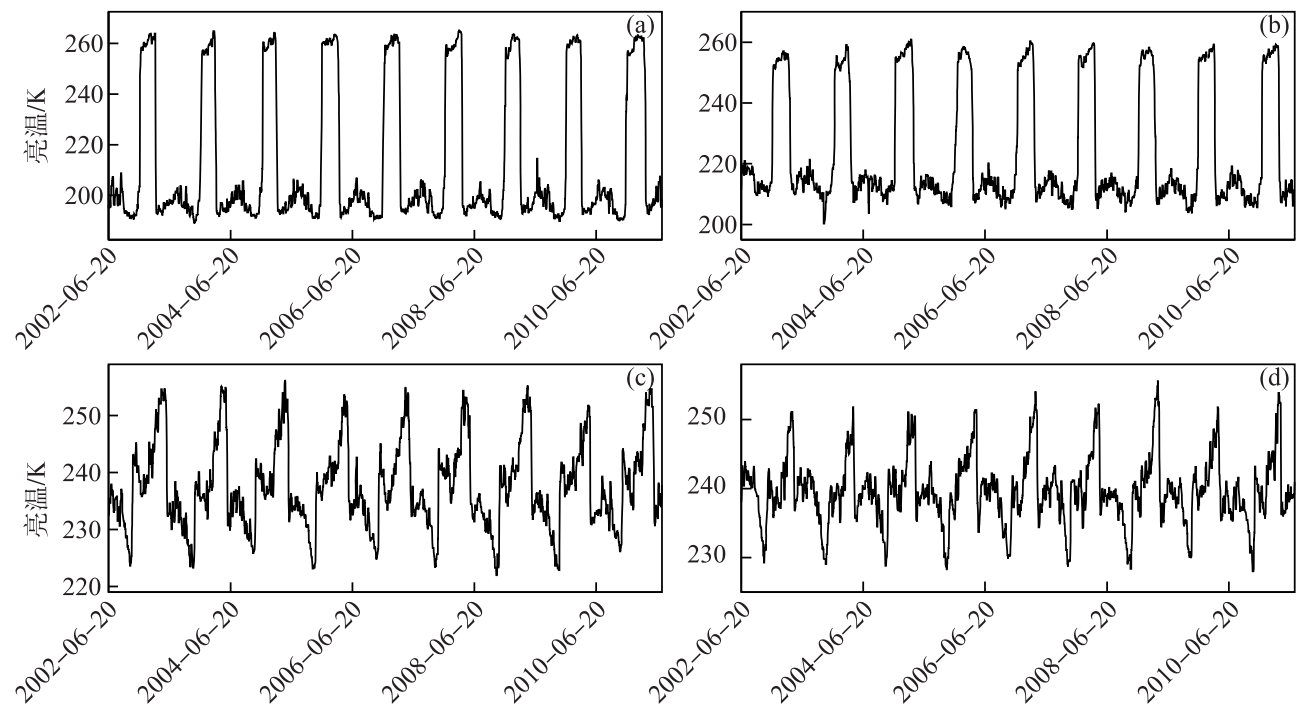

图 4 AMSR-E 传感器的 4 个湖泊亮温曲线 ( $\mathrm{a}$ : 青海湖; $\mathrm{b}$ :色林错; $\mathrm{c}$ : 哈拉湖; $\mathrm{d}$ : 阿其克库勒湖)

Fig.4 The TB curve of four lakes from the AMSR-E

(a: Lake Qinghai; b: Siling Co; c: Lake Hala; d: Lake Aqikekule)

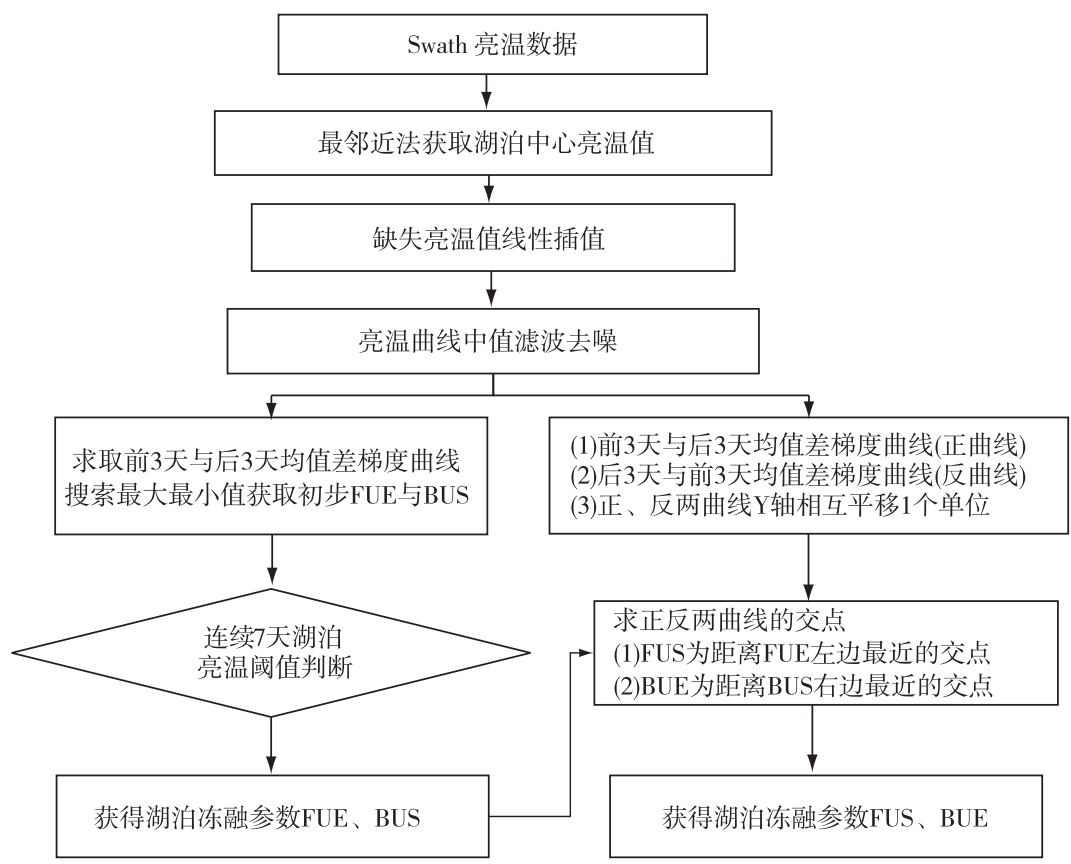

图 5 技术流程图

Fig.5 The technique flow chart

式中, $B T_{i}$ 代表湖泊第 $i$ 天亮温值, 且湖冰冻融参数 FUE 所处的月份 $M_{\mathrm{FUE}} \in(8 、 9 、 10 、 11 、 12 、 1)$, BUS 所处 的月份 $M_{\mathrm{BUS}} \in(2,3 、 4 、 5 、 6 、 7)$.

阈值法利用预处理后的湖泊亮温, 将前 3 天的亮温值总和与后 3 天的亮温值总和做差值, 考虑到冻结时 


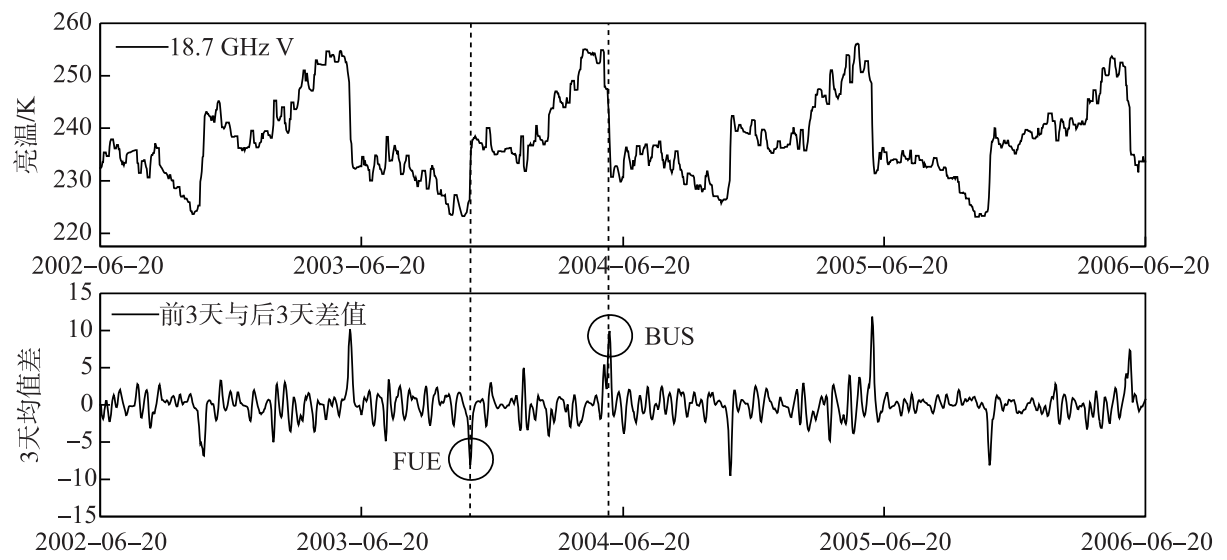

图 6 哈拉湖 FUE 与 BUS 参数获取

Fig.6 The FUE and BUS parameters of Lake Hala

会有冰厚度的变化 ${ }^{[38]}$, 经过测试以 $15 \mathrm{~K}$ 作为 “完全冻结” 参数的变化阈值 $t$ 的值, 而考虑到冰融化时环境的 复杂性 ${ }^{[42]}$, “开始融化” 参数的阈值 $t$ 为 $20 \mathrm{~K}$. 大于阈值记为 0 , 小于阈值记为 1 , 通过对前后 3 天阈值进行判 别并累加, 若累加值小于或等于 4 , 即所求的 “完全冻结” 参数能够满足 $T \leqslant \frac{4}{7}$; 而 “开始融化” 参数的累加 值需要大于等于 3 , 即 $T \geqslant \frac{3}{7}$, 则取该冻融周期内的最小值所对应的日期为湖冰完全冻结日, 最大值为开始 消融日. 湖泊变化动态阈值法 ${ }^{[39-40]}$ 的数学表达公式为:

$$
T=\frac{1}{2 n+1} \sum_{i=i-n}^{i=i+n} D_{i}, D= \begin{cases}1 & S_{i}<t \\ 0 & S_{i}>t\end{cases}
$$

式中, $i$ 表示一个冻融周期内的第 $i$ 天; $S_{i}$ 为第 $i$ 天的亮温值; $t$ 为湖冰物候的亮温阈值; $2 n+1$ 为窗口值, 即 7 天, 则 $n=3$.

若不满足上述条件,则需目视判读获取日期. 由图 4 可知,在 1 个冻融时间段内,湖泊亮温曲线呈 “几” 字型, 所以, 对不满足条件的冻融日期需要目视判定其是否处于 “几”字凸出部分的边缘. 基于上述最大、最 小值搜索法以及阈值法,可以得到湖冰“完全冻结”和“开始融化”两个参数.

b) FUS、BUE 湖冰冻融参数获取

当完全冻结 (FUE) 与开始融化 (BUS) 参数求出后, 以此为参考点求取 FUS 和 BUE 参数. 通过计算每天 与之前 3 天的亮温平均值和与之后 3 天的亮温平均值并做差值, 获得两条正反相交的差值曲线, 再通过正/ 反曲线相互间在 $\mathrm{Y}$ 轴平移 1 个单位, 求出距离已知 FUE 和 BUS 冻融参数最近的交点即为 FUS 与 BUE 冻融 参数. 如图 7 所示 FUS 为 FUE 左边第 1 个交点, BUE 为 FUS 右边第 1 个交点.

前 3 天与后 3 天差值 (正曲线) 求取公式:

$$
\left(B T_{i}+B T_{i-1}+B T_{i-2}+B T_{i-3}\right) / 4-\left(B T_{i}+B T_{i+1}+B T_{i+2}+B T_{i+3}\right) / 4 \pm 1
$$

后 3 天与前 3 天差值 (反曲线) 求取公式:

$$
\left(B T_{i}+B T_{i+1}+B T_{i+2}+B T_{i+3}\right) / 4-\left(B T_{i}+B T_{i-1}+B T_{i-2}+B T_{i-3}\right) / 4 \pm 1
$$

\section{3 结果与精度验证}

\section{1 青海湖实地观测数据验证}

选取青海湖 2002-2006 年的湖冰冻融地面观测数据 ${ }^{[28]}$ 与 AMSR-E 微波数据提取的湖冰冻融日期进行 对比 (表 1), 可知被动微波数据所监测的冻融日期与地面观测的冻融日期相比误差较小, 在 2002-2006 年 间 FUE 参数最大误差仅为 3 天, BUS 参数最大误差仅为 2 天. 


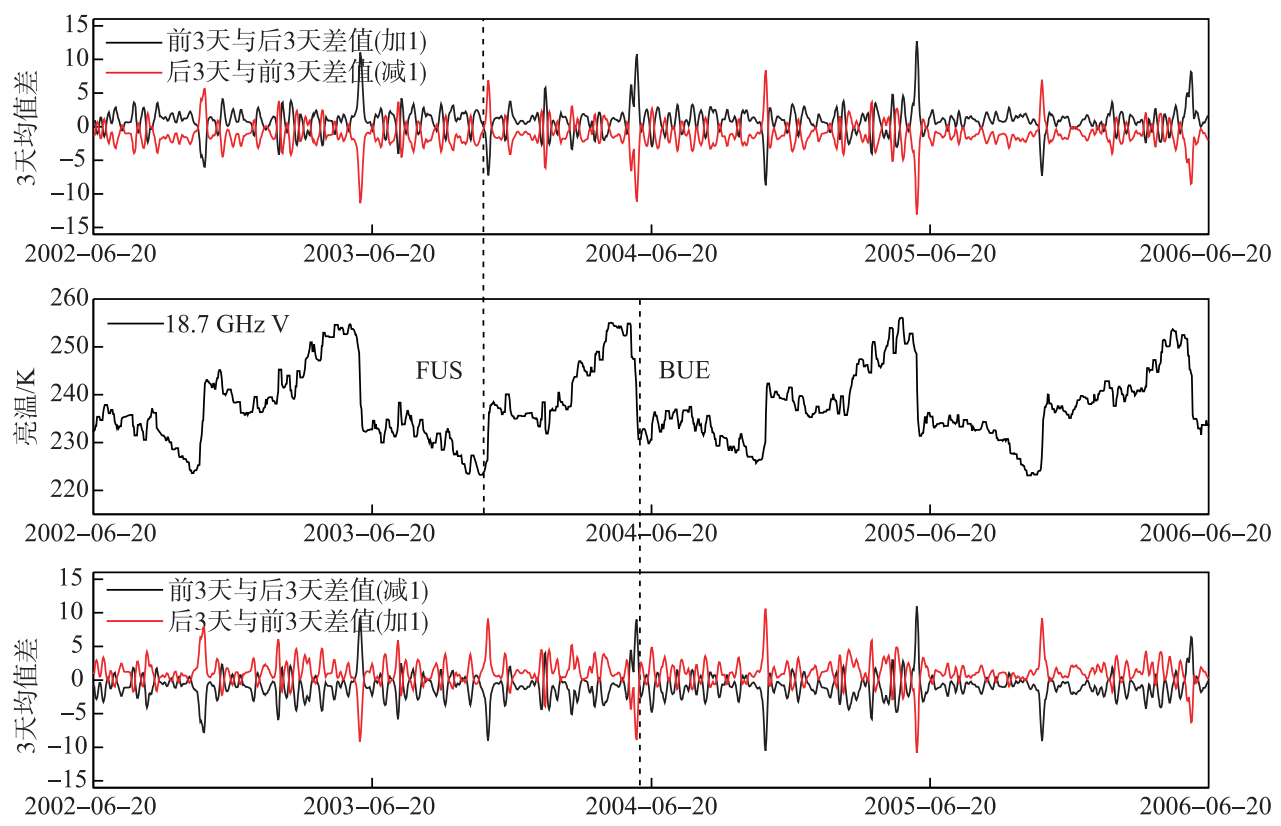

图 7 哈拉湖 FUS 与 BUE 参数获取

Fig.7 The FUS and BUE parameters of Lake Hala

表 1 青海湖 2002-2006 年 FUE/BUS 地面观测数据验证

Tab.1 The accuracy verification of Lake Qinghai's FUE/BUS with in-situ data(2002-2006)

\begin{tabular}{|c|c|c|c|}
\hline \multicolumn{2}{|c|}{ 完全冻结日期 (年-月-日) } & \multicolumn{2}{|c|}{ 开始融化日期 ( 年-月-日) } \\
\hline 微波监测 & 地面观测 & 微波监测 & 地面观测 \\
\hline $2002-12-25$ & $2002-12-26$ & & \\
\hline $2003-12-29$ & $2003-12-27$ & $2003-03-29$ & $2003-03-31$ \\
\hline $2004-12-30$ & $2004-12-30$ & $2004-03-21$ & $2004-03-20$ \\
\hline $2005-12-20$ & $2005-12-19$ & $2005-03-26$ & $2005-03-26$ \\
\hline $2006-12-20$ & $2006-12-23$ & $2006-04-04$ & $2006-04-04$ \\
\hline
\end{tabular}

\subsection{MODIS 无云光学遥感验证}

由于青海湖水文站的观测数据只有青海湖的 FUE 与 BUS 记录, 没有 FUS 与 BUE 记录, 故本文利用中 等分辨率的 MODIS 逐日无云积雪面积数据从光学遥感观测的方面对被动微波辐射计提取的 4 个湖冰物候 参数进行验证. 光学影像对湖冰冻融研究一般将 $10 \%$ 和 $90 \%$ 的水体面积作为临界值判别湖冰的冻、融情 况 $^{[8,23]}$. 通过利用该國值法从 MODIS 逐日无云积雪面积数据中获得的 4 个湖泊的冻融参数与被动微波辐射 计数据获取的冻融参数相比 (图 8), 发现 4 个湖泊 FUS、FUE、BUS 和 BUE 的 $R^{2}$ 分别为 $0.9867 、 0.9680$ 、 0.9651 和 0.9732 . 均方根误差 ( RMSE) 分别为 2.2889、3.5744、4.6225 和 4.0370 天. 而其中尤以 FUS 的 RMSE 最小, 仅 2.3 天, 而 BUS 的 RMSE 最大, 为 4.6 天. 由于 MODIS 逐日无云积雪面积数据在个别时段存在 $<50 \%$ 的不确定性,这也导致了 RMSE 较大.

\section{3 误差来源分析}

在开展湖冰物候监测过程中, 主要的误差来源分为两方面:一方面为亮温数据获取及其处理过程, 另一 方面为湖泊所处的环境变化.

由于星载被动微波的瞬时视场角为 $27 \mathrm{~km} \times 16 \mathrm{~km} / 22 \mathrm{~km} \times 14 \mathrm{~km}$, 当湖泊面积较小或形状不规整时, 所获 

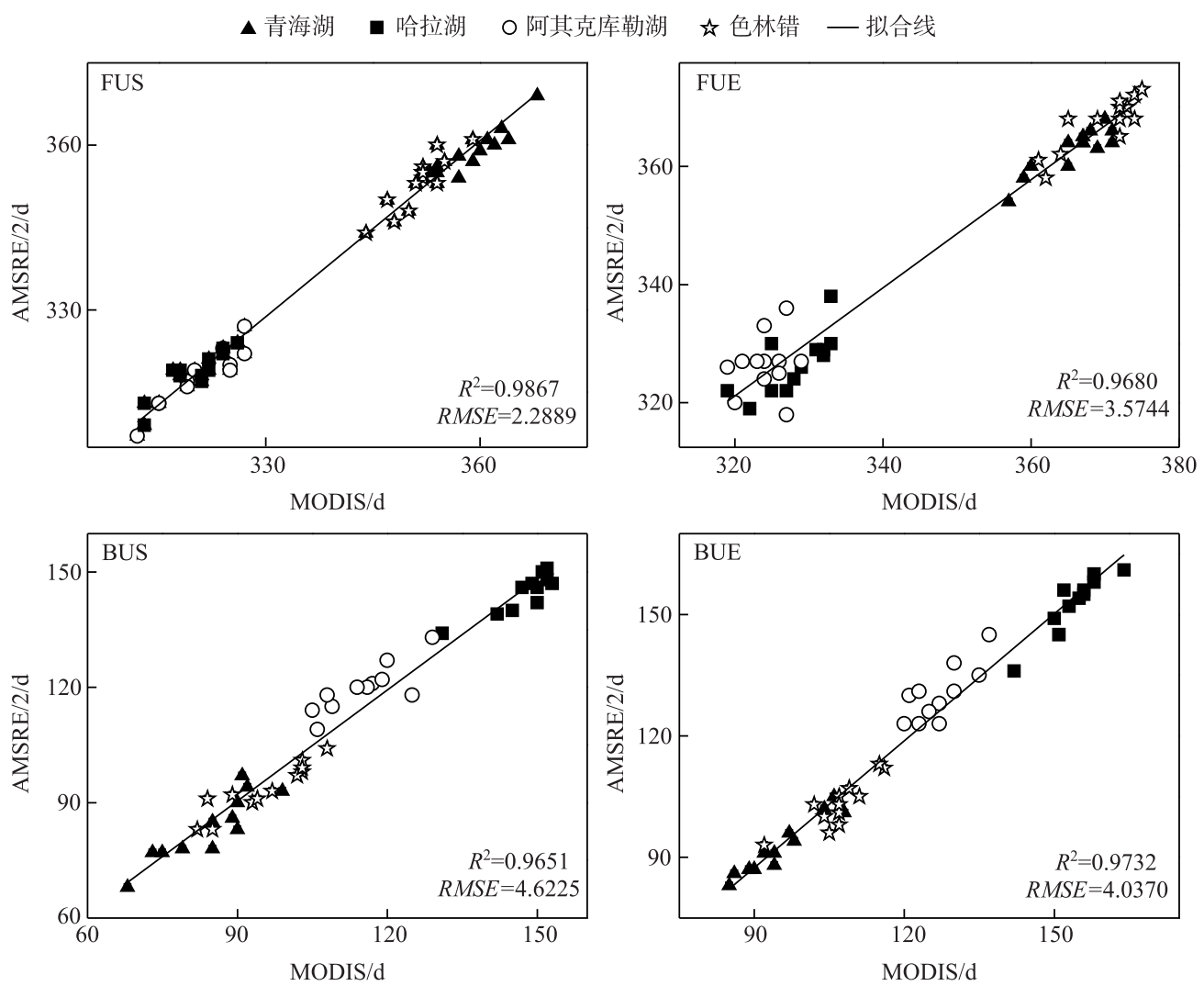

图 8 MODIS 湖冰冻融参数验证图

Fig.8 The accuracy verification of lake ice phenology with MODIS

取的湖泊亮温受到湖岸辐射的影响, 从而导致混合像元问题, 如哈拉湖、阿其克库勒湖就明显受到湖岸的影 响; 此外, 在获取湖泊信息时只是获取中心区域的 $432 \mathrm{~km}^{2} / 308 \mathrm{~km}^{2}$ 面积范围的一个微波像元信号, 无法获 取整个湖泊的信息, 而湖冰的冻结与融化在一般情况下都是由湖岸向湖心发生变化,故会存在一定的误差; 湖泊信号获取过程中, 为了弥补数据缺失以及消除噪声信息, 对原始湖泊亮温信号做插值处理和中值平滑 处理等预处理,因此可能造成部分信息失真, 导致对湖冰物候参数提取时误差扩大.

湖泊所处环境的变化也会对湖冰物候的监测造成误差, 除了受湖面温度的影响外, 在非冰冻期, 风的吹 动引起湖泊波浪起伏, 改变湖面粗精度, 直接影响湖水的发射率; 在冰冻期, 风将湖面冻结的薄冰吹散, 露出 冰层下的水体, 微波亮温会发生快速变化. 因此, 冰冻期内湖泊反复冻结融化, 造成亮温信号变化不稳定, 致 使物候参数提取产生误差.

\section{4 结论}

湖冰是陆地淡水冰的主要组成部分, 湖冰物候对气候变化具有指示作用, 其历史观测资料可以反映湖 泊所处环境的气候变化. 本文围绕青藏高原湖冰物候, 利用被动微波轨道亮温数据, 开展有关湖冰物候监测 方法的研究. 通过对青藏高原湖冰物候监测方法研究及精度验证, 获得以下认识:

被动微波 $18.7 \mathrm{GHz}$ 垂直极化亮温数据分辨率低但对冰水相变敏感, 而被动微波轨道亮温数据相较 EASE-Grid 重采样数据能更精确获得湖泊亮温信号. 因此, 基于被动微波轨道( Swath) 亮温数据, 可获得亚像 元级 (AMSR-E $/ 2: 432 \mathrm{~km}^{2} / 308 \mathrm{~km}^{2}$ ) 湖泊亮温数据, 且湖泊亮温在长时间序列变化上具有明显的特征, 可反 映湖冰物候的信息,为湖冰物候信息的提取提供数据源. 
通过将 4 天最大值、最小值搜索法与阈值判别法结合, 可减小人工解译所带来的误差, 弱化湖冰物候判 别时所受的冰厚度变化和外界因素的影响, 提高湖冰物候信息的获取速度; 基于被动微波轨道亮温数据与 阈值判别法获取的青海湖、色林错、哈拉湖与阿其克库勒湖的湖冰物候参数, 经验证,算法对 FUS、FUE、BUS 与 BUE 4 个湖冰物候参数监测的误差控制在 2 4 天内,可有效进行湖冰物候监测.

湖冰物候参数提取虽存在误差, 与传统光学数据的监测精度相比,微波监测精度已有很大提高, 有望实 现更大范围内更多湖泊湖冰物候的监测. 在青藏高原地区与阿其克库勒湖 $\left(355.6 \mathrm{~km}^{2}\right)$ 面积大小相近的湖 泊约有 30 个 ${ }^{[33]}$, 而且在蒙古高原、欧亚大陆中部也存在大量有结冰现象的湖泊. 该方法有望实现青藏高原 地区等大范围内湖冰物候的监测, 补充这些地区的数据空缺, 实现湖冰物候的应用价值. 此外, 在湖冰冻融 变化过程中存在湖冰厚度的变化 ${ }^{[38]}$, 而湖冰厚度变化对冰上运输、渔业捕捞等经济活动有重要影响, 因此, 要更清晰认识湖冰厚度的变化,有待进一步研究.

致谢: 感谢美国国家冰雪数据中心(National Snow and Ice Data Center) 提供 AMSR-E L2A Swath 亮温数据, 感 谢日本宇宙航空研究开发机构 (Japan Aerospace Exploration Agency) 提供 AMSR-2 L1R Swath 亮温数据, 感谢 中国国家卫星气象中心提供 MWRI Swath 亮温数据; 感谢 Science Data Bank 提供青藏高原 MODIS 逐日无云 积雪面积数据集数据.

\section{5 参考文献}

[ 1 ] O'Reilly CM, Sharma S, Gray DK et al. Rapid and highly variable warming of lake surface waters around the globe. Geophysical Research Letters, 2015, 42(24). DOI: 10.1002/2015GL066235.

[ 2 ] Zhang G, Yao T, Xie H et al. Estimating surface temperature changes of lakes in the Tibetan Plateau using MODIS LST data. Journal of Geophysical Research Atmospheres, 2015, 119(14): 8552-8567.

[ 3 ] Kropácek J, Maussion F, Chen F et al. Analysis of ice phenology of lakes on the Tibetan Plateau from MODIS data. The Cryosphere, 2013, 7(1): 287.

[ 4 ] He YQ, Zhang ZL, Yao TD et al. Modern changes of the climate and glaciers in China's monsoonal temperate-glacier region 2003, 4: 550-558. [何元庆, 张忠林, 姚檀栋等. 中国季风温冰川区近代气候变化与冰川动态. 地理学报, $2003,4: 550-558$.

[ 5 ] Kouraev AV, Semovski SV, Shimaraev MN et al. Observations of Lake Baikal ice from satellite altimetry and radiometry. Remote Sensing of Environment, 2007, 108(3) : 240-253.

[ 6 ] Lehner B, Döll P. Development and validation of a global database of lakes, reservoirs and wetlands. Journal of Hydrolo$g y, 2004,296(1): 1-22$.

[ 7 ] Liu XD, Chen BD. Climatic warming in the Tibetan Plateau during recent decades. International Journal of Climatology, 2015, 20 (14) : 1729-1742.

[ 8 ] Yao TD, Guo XJ, Thompson L et al. Delta O-18 record and temperature change over the past 100 years in ice cores on the Tibetan Plateau. Science in China Series D-Earth Sciences, 2006, 36(1): 1-8. [姚檀栋, 郭学军, Lonnie Thompson 等. 青藏高原冰芯过去 100 年 $\delta^{18} \mathrm{O}$ 记录与温度变化. 中国科学: 地球科学, 2006, 36(1) : 1-8.]

[ 9 ] Cheng G, Wu T. Responses of permafrost to climate change and their environmental significance, Qinghai-Tibet Plateau. Journal of Geophysical Research Atmospheres, 2007, 112(F2) : 93-104.

[10] Wan W, Long D, Hong Y et al. A lake data set for the Tibetan Plateau from the 1960s, 2005, and 2014. Scientific Data, 2016, 3: 160039. DOI: 10.1038/sdata.2016.39.

[11] Ma RH, Yang GS, Duan HT et al. China's lakes at present: number, area and spatial distribution. Science China Earth Sciences, 2011, 54(2): 283-289.

[12] Zhang G, Yao T, Xie H et al. Lakes' state and abundance across the Tibetan Plateau. Chinese Science Bulletin, 2014,59 (24) : 3010-3021.

[13] Yao XJ, Liu SY, Li L et al. Spatial-temporal variations of lake area in Hoh Xil region in the past 40 years. Acta Geographica Sinica, 2013, 68(7): 886-896. [姚晓军, 刘时银, 李龙等. 近 40 年可可西里地区湖泊时空变化特征. 地理学 报, 2013, 68(7): 886-896.]

[14] Zhang X, Wu YH, Zhang X. Water level variation of inland lakes on the south-central Tibetan Plateau in 1972-2012. Acta 
Geographica Sinica, 2014, 69(7) : 993-1001. [张金金, 吴艳红, 张金金. 1972-2012 年青藏高原中南部内陆湖泊的水位 变化. 地理学报, 2014, 69(7): 993-1001.]

[15] Liu Z, Zhao J, Shi YF et al. The relationship between the main lakes and the climate change in Xinjiang (2000-2009) by using MODIS. Journal of Arid Land Resources \& Environment, 2011, 25(10)：155-160. [刘吉, 赵军, 师银芳等. 利用 MODIS 数据对 2000 2009 年新疆主要湖泊面积变化与气候响应的分析. 干旱区资源与环境, 2011, 25(10): 155-160.]

[16] Zhang G, Xie H, Kang S et al. Monitoring lake level changes on the Tibetan Plateau using ICES at altimetry data (20032009). Remote Sensing of Environment, 2011, 115(7): 1733-1742.

[17] Laba ZM, Deji YZ, Laba et al. Remote sensing analysis on the area variations of Tangra Yutso in Tibetan Plateau over the past 40 years. $J$ Lake $S c i, 2017,29(2)$ : 480-489. [拉巴卓玛, 德吉央宗, 拉巴等. 近 40 a 西藏那曲当惹雍错湖泊面 积变化遥感分析. 湖泊科学, $2017,29(2): 480-489$.

[18] Zhang G, Yao T, Shum CK et al. Lake volume and groundwater storage variations in Tibetan Plateau's endorheic basin. Geophysical Research Letters, 2017, 44: 5550-5560.

[19] Preston DL, Caine N, Mcknight DM et al. Climate regulates alpine lake ice cover phenology and aquatic ecosystem structure. Geophysical Research Letters, 2016, 43: 5353-5360. DOI: 10.1002/2016GL069036.

[20] O'Reilly CM, Sharma S, Gray DK et al. Rapid and highly variable warming of lake surface waters around the globe. Geophysical Research Letters, 2015, 42. DOI: 10.1002/2015GL066235.

[21] Chen XZ, Wang GY, Li WJ et al. Lake ice and its remote sensing monitoring in the Tibetan Plateau. Journal of Glaciolgy \& Geocryology, 1995, 17(3) : 241-246. [陈贤章, 王光宇, 李文君等. 青藏高原湖冰及其遥感监测. 冰川冻土, $1995,17(3): 241-246$.

[22] Yin QJ, Yang YL. Remote sensing monitoring of Lake Qinghai based on EOS/MODIS data. J Lake Sci, 2005, 17 (4) : 356-360. DOI : 10.18307/2005.0413. [ 殷青军, 杨英莲. 基于 EOS/MODIS 数据的青海湖遥感监测. 湖泊科学, 2005, 17(4) : 356-360.]

[23] Kropácek J, Maussion F, Chen F et al. Analysis of ice phenology of lakes on the Tibetan Plateau from MODIS data. Cryosphere, 2013, 7(1) : 287-301.

[24] Yao XJ, Li L, Zhao J et al. Spatial-temporal variations of lake ice in the Hoh Xil region from 2000 to 2011. Journal of Geographical Science, 2015, 70(7): 1114-1124. [姚晓军, 李龙, 赵军等. 近 10 年来可可西里地区主要湖泊冰情时空 变化. 地理学报, 2015, 70(7): 1114-1124.]

[25] Gou P, Ye QH, Wei QF. Lake ice change at the Nam Co Lake on the Tibetan Plateau during 2000-2013 and influencing factors. Progress in Geography, 2015, 34(10): 1241-1249. [勾鹏, 叶庆华, 魏秋方. 2000-2013 年西藏纳木错湖冰 变化及其影响因素. 地理科学进展, 2015, 34(10): 1241-1249.]

[26] Huang XD, Hao XH, Wang W et al. Algorithms for cloud removal in MODIS daily snow products. Journal of Glaciology \& Geocryology, 2012, 34(5) : 1118-1126. [ 黄晓东, 郝晓华, 王玮等. MODIS 逐日积雪产品去云算法研究. 冰川冻土, 2012, 34(5) : 1118-1126. ]

[27] Yu J, Zhang G, Yao T et al. Developing daily cloud-free snow composite products from MODIS Terra-Aqua and IMS for the Tibetan Plateau. IEEE Transactions on Geoscience \& Remote Sensing, 2016, 54(4) : 2171-2180.

[28] Che T, Li X, Jin R. Monitoring the frozen duration of Qinghai Lake using satellite passive microwave remote sensing low frequency data. Chinese Sci Bull, 2009, 54(6): 787-791. [车涛, 李新, 晋锐. 利用被动微波遥感低频亮温数据监测 青海湖封冻与解冻期. 科学通报, 2009, 54(6) : 787-791.]

[29] Tao AQ. Research on the variation of Namco Lake ice by passive microwave remote sensing [Dissertation]. Nanjing: Nanjing University, 2014. [ 陶安琪. 被动微波遥感纳木错湖冰变化研究 [学位论文]. 南京: 南京大学, 2014.]

[30] Brodzik MJ, Billingsley B, Haran T et al. EASE-Grid 2.0: Incremental but significant improvements for earth-gridded data sets. ISPRS International Journal of Geo-Information, 2012, 1(3) : 32-45.

[31] Wu SH, Yin YH, Zheng D et al. Climate changes in the Tibetan Plateau during the last three decades. Acta Geographica Sinica, 2005, 60(1) : 3-11. [ 吴绍洪, 尹云鹤, 郑度等. 青藏高原近 30 年气候变化趋势. 地理学报, 2005, 60(1): 3-11.]

[32] Preston DL, Caine N, Mcknight DM et al. Climate regulates alpine lake ice cover phenology and aquatic ecosystem structure. Geophysical Research Letters, 2016, 43. DOI: 10.1002/2016GL069036. 
[33] Lehner B, Döll P. Development and validation of a global database of lakes, reservoirs and wetlands. Journal of Hydrolo$g y, 2004,296(1 / 2 / 3 / 4): 1-22$.

[34] Huang D, Qiu YB, Shi LJ et al. Cross-calibration for passive microwave measurement of MWRI and AMSR-E. Science of Surveying and Mapping, 2017, 42(1): 136-142. [黄端, 邱玉宝, 石利娟等. 两种星载微波辐射计被动亮温数据的 交叉定标. 测绘科学, 2017, 42(1): 136-142.]

[35] Qiu YB, Guo HD, Shi LJ et al. Global land surface emissivity dataset based on AMSR-E observations. Remote Sensing Technology and Application, 2016, 31(4) : 809-819. [邱玉宝, 郭华东, 石利娟等. 基于 AMSR-E 的全球陆表被动微 波发射率数据集. 遥感技术与应用, 2016, 31(4): 809-819.]

[36] Du J, Kimball JS, Duguay C et al. Satellite microwave assessment of Northern Hemisphere lake ice phenology from 2002 to 2015. The Cryosphere, 2017, 11: 47.

[37] Qiu YB, Guo HD, Chu D et al. MODIS daily cloud-free snow cover product over Tibetan Plateau (2002 to 2015). ScienceDB. DOI: 10.11922/sciencedb.170.55.

[38 ] Kang KK, Duguay CR, Lemmetyinen J et al. Estimation of ice thickness on large northern lakes from AMSR-E brightness temperature measurements. Remote Sensing of Environment, 2014, 150(7) : 1-19.

[39] Qu B, Kang SC, Chen F et al. Lake ice and its effect factors in the Nam Co Basin, Tibetan Plateau. Progressus Inquisitiones De Mutatione Climatis, 2012, 8(5) : 327-333. [曲斌, 康世昌, 陈锋等. 2006-2011 年西藏纳木错湖冰状况及 其影响因素分析. 气候变化研究进展, 2012, 8(5):327-333.]

[40] Latifovic R, Pouliot D. Analysis of climate change impacts on lake ice phenology in Canada using the historical satellite data record. Remote Sensing of Environment, 2007, 106(4) : 492-507.

[41] Robertson DM, Ragotzkie RA, Magnuson JJ. Lake ice records used to detect historical and future climatic changes. Climatic Change, 1992, 21(4) : 407-427. 